\title{
ISCHEMIC POSTCONDITIONING REDUCES INFARCT SIZE THROUGH THE $\alpha-1$ ADRENERGIC RECEPTOR PATHWAY
}

\author{
Bruno Buchholz ${ }^{a * 1}$, Verónica D’Annunzio ${ }^{a * 2}$, Jorge F Giani ${ }^{b}$, Nadezda Siachoque ${ }^{a}$, \\ Fernando P Dominici ${ }^{\mathrm{b} 2}$, Daniel Turyn ${ }^{\mathrm{b} 2}$, Virginia Perez ${ }^{\mathrm{a}}$, Martín Donato ${ }^{\mathrm{a} 2}$, Ricardo J. Gelpi ${ }^{\mathrm{a} 2}$
}

a: Institute of Cardiovascular Physiopathology, Department of Pathology, School of Medicine, University of Buenos Aires, Argentina. JE Uriburu 950 - 2nd floor. (1114), Buenos Aires - Argentina. b: Institute of Chemistry and Biological Physical Chemistry, School of Pharmacy and Biochemistry, University of Buenos Aires, Buenos Aires, Argentina. Junín 954 - 2nd floor. (1113). Buenos Aires - Argentina.

1: Post Doctoral fellow of the National Council of Scientific and Technological Research (CONICET)

${ }^{2}$. Members of the National Council of Scientific and Technological Research (CONICET)

* First and second authors contributed equally to this manuscript.

Short running title: $\alpha-1$ adrenergic receptor in postconditioning

Corresponding author: Ricardo J. Gelpi: Institute of Cardiovascular Physiopathology, Pathology Department, Faculty of Medicine, University of Buenos Aires, Argentina. JE Uriburu 950 - 2nd floor. C1114AAD, Buenos Aires - Argentina.Tel/Fax: 54-11-4962-4945. E-mail: rgelpi@fmed.uba.ar 


\section{ABSTRACT}

The $\alpha 1$-adrenergic receptors ( $\alpha 1-A R s)$ are involved in preconditioning. Given that certain intracellular pathways appear to be shared by preconditioning and postconditioning, it is possible that postconditioning could be also mediated by a1-ARs. The objective was to evaluate, by analyzing infarct size, if $\alpha 1-A R s$ activation could trigger postconditioning; and

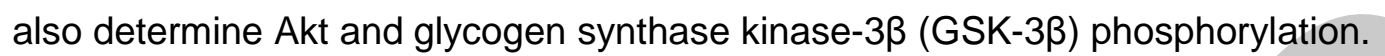
Langendorff-perfused rat hearts were subjected to 30 minutes of ischemia and 120 minutes of reperfusion $(I / R ; n=8)$. After 30 min of global ischemia, we performed 6 cycles of reperfusion/ischemia of $10 \mathrm{sec}$ each, followed by $120 \mathrm{~min}$ of reperfusion (postcon; $n=9$ ). In another postcon group, we administered prazosin during postcon protocol (postcon + prazosin; $n=7$ ). Finally, we repeated the I/R group but prazosin (prazosin; $n=7$ ), phenylephrine (PE; $n=5)$ and clonidine $(C L ; n=6)$, were administered during the first 2 minutes of reperfusion. Infarct size was measured using the triphenyltetrazolium chloride technique. Total and phosphorylated Akt and mitochondrial GSK-3ß expression were measured by Western blot. Infarct size was $58.1 \pm 5.1 \%$ in $\mathrm{I} / \mathrm{R}$. Postcon and PE reduced infarct size to $40.1 \pm 2.9 \%$ and $35.3 \pm 5.5 \%$ respectively ( $p<0.05$ vs. $l / R$ ). Postcon + prazosin administration abolished the beneficial effect on infarct size $(61.6 \pm 4.5 \% ; p<0.05$ vs. postcon). Cytosolic Akt phosphorylation and mitochondrial GSK-3ß phosphorylation were higher in the postcon and PE groups compared with the $\mathrm{I} / \mathrm{R}$ and postcon + prazosin groups. Prazosin or clonidine administration did not modify neither protein expression nor infarct size. Our data demonstrate that postconditioning decrease infarct size by activation of the a1-AR pathway through Akt and GSK-3ß phosphorylation.

Keywords: Ischemic postconditioning, $\alpha-1$ adrenergic receptor, infarct size, Akt, glycogen synthasa kinase $3 \beta$ 


\section{INTRODUCTION}

Brief intermittent periods of ischemia and reperfusion performed before long ischemia (termed ischemic preconditioning), have demonstrated protection of the myocardium against ischemia/reperfusion injury ${ }^{1}$. This cardioprotective strategy has limited clinical application in patients with acute myocardial infarction due to the inability to predict the moment of coronary artery occlusion. The concept of ischemic postconditioning may be useful, as it has been described as an entity by which brief periods of ischemia/reperfusion, performed at the onset of reperfusion, reduce infarct size ${ }^{2}$.

Different authors have provided evidence that the reduction on infarct size conferred by postconditioning could be triggered by activation of multiple types of cell membrane receptors which include adenosine $\mathrm{A}_{1}{ }^{3}$, bradykinin $\mathrm{B}_{2}{ }^{4}$ and opioids ${ }^{5}$. However, other types of membrane receptors that could be involved in the postconditioning mechanism are the $\alpha$-adrenergic receptors ( $\alpha 1-A R s)$. The beneficial effects of preischemic $\alpha$-ARs activation were shown by Gao et al. ${ }^{6}$, who found that activation of $\alpha 1-A R s$ maintains cytosolic and mitochondrial $\mathrm{Ca}^{2+}$ homeostasis, improves contraction, and preserves ATP content under simulated ischemia/reperfusion. Studies by Banerjee et al. ${ }^{7}$ concluded that transient ischemic preconditioning is mediated by sympathetic neurotransmitter release and $\alpha-1-A R s$ stimulation. Conversely, Vasara et al. ${ }^{8}$ suggested that $\alpha 1-A R s$ stimulation mimics ischemic preconditioning, but that it is not an essential component of the mechanism. However, there is no evidence of the possible role of $\alpha 1-A R s$ as a trigger for postconditioning. Given that certain intracellular pathways, recruited at the time of myocardial reperfusion and appearing to be shared by both ischemic preconditioning and postconditioning, we hypothesized that activation of $\alpha 1-A R s$ at the time of reperfusion is an important part of the mechanism of ischemic postconditioning.

Previous studies identified the survival kinases phosphatidylinositol 3-kinase (PI3K)-Akt as key players in the protection conferred by postconditioning ${ }^{9}$. However, studies evaluating the postconditioning mechanism did not consider the possibility of Akt phosphorylation via a $\alpha 1-A R$ pathway in this protective mechanism. The inactivation of

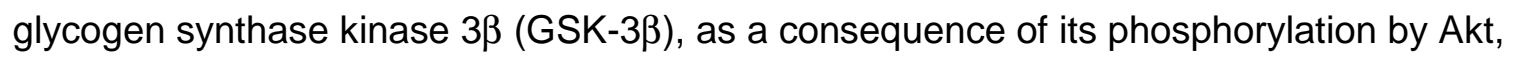
has been implicated in the ischemic preconditioning mechanism ${ }^{10}$ and in postconditioning ${ }^{11}$. However, the effect of $\alpha 1$-ARs stimulation as part of the mechanism of 
ischemic postconditioning on Akt and GSK-3 $\beta$ expression and in phosphorylation, is unknown. Thus, the aim of this study is to evaluate, by analyzing infarct size, if a1-ARs may trigger postconditioning. Since it's known that Akt and GSK-3 $\beta$ phosphorylation participate in the protection mechanism of ischemic postconditioning, a second objective was to determine if the reduction of the infarct size by the induced activation of $\alpha 1-A R s$ requires Akt and GSK-3 $\beta$ phosphorylation.

\section{MATERIAL AND METHODS}

Wistar male rats (200 to $250 \mathrm{gr}$ ) were sacrificed with an intraperitoneal injection of pentobarbital $(150 \mathrm{mg} / \mathrm{kg})^{12}$. The heart was excised from the animals and placed in a perfusion system according to the Langendorff technique. All hearts were perfused with a Krebs-Henseleit buffer containing $\mathrm{NaCl} 118.5 \mathrm{mM}, \mathrm{KCl} 4.7 \mathrm{mM}, \mathrm{NaHCO}_{3} 24.8 \mathrm{mM}$, $\mathrm{KH}_{2} \mathrm{PO}_{4} 1.2 \mathrm{mM}, \mathrm{Mg} \mathrm{SO}_{4} 1.2 \mathrm{mM}, \mathrm{CaCl}_{2} 1.8 \mathrm{mM}$ and glucose $10 \mathrm{mM}$, pH 7.2 - 7.4, and gassed with $95 \% \mathrm{O}_{2}-5 \% \mathrm{CO}_{2}$ at $37^{\circ} \mathrm{C}$. Two electrodes were sutured and connected to a pacemaker with a constant heart rate of 275 beats per minute (bpm). The experiments were approved by the Animal Care and Research Committee of the University of Buenos Aires (Protocol \# 2948/2010). All the procedures were performed in compliance with the guidelines of the American Physiological Society "Guiding Principles for the Care and Use of Vertebrate Animals in Research and Training."

A latex balloon connected to a pressure transducer (Deltram II, Utah Medical System) via a polyethylene cannula was inserted into the left ventricle (LV) for measurement of left ventricular pressure. The latex balloon was filled with water to achieve a left ventricular end diastolic pressure (LVEDP) of 8-10 mmHg. In addition, coronary perfusion pressure (CPP) was recorded using a pressure transducer connected to the perfusion line. All hearts were perfused at a constant flow. Coronary flow was adjusted to obtain a CPP of $70.5 \pm 4.2 \mathrm{mmHg}$ during the initial stabilization period. This flow level was maintained constant throughout the experiment.

\section{Experimental Protocols:}


Ischemia/reperfusion group (I/R; $n=8)$ : Myocardial infarction was induced by 30 minutes of global no-flow ischemia followed by 120 minutes of reperfusion. Global no-flow ischemia was induced by abruptly decreasing the total coronary flow provided by the perfusion pump.

Ischemic postconditioning group (postcon; $n=9$ ): The same protocol as in I/R group was repeated, but at the onset of reperfusion we performed 6 cycles of $10 \mathrm{sec}$ of reperfusion followed by $10 \mathrm{sec}$ of ischemia ( 2 minutes total intervention, ischemic postconditioning protocol). Reperfusion was then continued for 120 minutes.

Ischemic postconditioning + prazosin group (postcon+prazosin; $n=7)$ : The same protocol as postcon was followed except for the administration of Prazosin Hydrochloride (Sigma) ( $\alpha 1-\mathrm{ARs}$ blocker), diluted in isotonic saline solution at a $1 \mu \mathrm{M}$ during ischemic postconditioning. It's important to remark that prazosin was administered simultaneously while performing the postconditioning protocol. It was dissolved in the Krebs solution during the 6 cycles of short ischemia/ reperfusion (10 sec each). Once the postconditioning protocol was performed, hearts were perfused solely with KrebsHenseleit solution until the protocol was complete.

Prazosin group ( $n=7)$ : The same protocol was followed as in $I / R$, except for the administration of Prazosin Hydrochloride (Sigma) at a $1 \mu \mathrm{M}$ concentration during the entire first 2 minutes of the reperfusion period. This was performed in order to rule out the effects of the drug itself on myocardial viability.

Phenylephrine group (PE; $n=5)$ : In order to assess if a1-ARs activation would trigger the postconditioning protection mechanism, we administered L-phenylephrine hydrochloride (Sigma), a selective a1-ARs agonist, diluted in isotonic saline solution at $50 \mu \mathrm{M}, 5 \mu \mathrm{M}$ and $50 \mathrm{nM}$ concentrations during 2 minutes at the beginning of reperfusion, (after 30 minutes of global ischemia). Reperfusion was then continued for $120 \mathrm{~min}$.

Clonidine group ( $C L ; n=6)$ : In this group we administered clonidine hydrochloride (Research Biochemicals International), a selective a2-ARs agonist, to confirm our hypothesis that $\alpha 1$-ARs receptors, and not $\alpha 2-A R s$, are the ones involved in myocardial protection.Clonidine was administered diluted in isotonic saline solution at a $10^{-6} \mathrm{M}$ concentration during the first two minutes of reperfusion. Again, this administration was 
performed after 30 minutes of global ischemia. Reperfusion was then continued for 120 $\min$.

Normoxic group ( $\mathrm{Nx} ; \mathrm{n}=4)$ : This group was only used as a control group for the Western blot determinations. Hearts were stabilized during $20 \mathrm{~min}$ and then left ventricle samples were taken and deep frozen at $-180^{\circ} \mathrm{C}$ for their further processing. All the experimental groups were repeated and LV samples were taken at $20 \mathrm{~min}$ of reperfusion for the Western blot determination ( $n=4$ per group). In the groups where the Western blot determinations were performed, the total perfusion time was of approximately $20 \mathrm{~min}$, similar to the normoxic group used as the control for Western blot.

\section{$\underline{\text { Infarct Size Measurement }}$}

Infarct size was evaluated using 2, 3, 5-Triphenyltetrazolium chloride. After 120 min of reperfusion, the hearts were deep frozen at $-20^{\circ} \mathrm{C}$ during 30 minutes and then cut into $2 \mathrm{~mm}$ transverse slices from apex to base. The sections were incubated for 20 minutes in a 1\% 2,3,5- Triphenyltetrazolium chloride (Sigma) solution diluted in Tris buffer

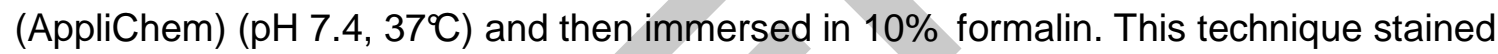
viable sections red whereas non-viable sections remained unstained. All the sections were scanned and planimetered (Image Pro Plus, version 4.5). Infarct size was expressed as a percentage of the left ventricular area.

Mitochondrial and Cytosolic Isolation and Fractioning:

Frozen samples were homogenized in 5 volumes of buffer MSHE (pH 7.6) with phosphatase and protease inhibitors (Halt (tm) Protease \& Phosphatase Inhibitor Cocktail EDTA-free form Pierce), using a Potter Elvehjem homogenizer. Homogenates were centrifuged at $657 \mathrm{G}$ for 10 minutes, filtered, and centrifuged again at 8,000 $\mathrm{G}$ for 10 minutes. Pellets were resuspended in MSHE buffer (0.21 M mannitol/0.07 M sucrose/10 mM Hepes, pH 7.4/1 mM EDTA/1 mM EGTA) with bovine serum albumin (BSA) and 30\% Percoll. Suspensions were centrifuged at $8,000 \mathrm{G}$ for 30 minutes at $4^{\circ} \mathrm{C}$. Mitochondrial fractions were separated and rinsed once with MSHE buffer with BSA at 10,509 G for 10 minutes at $4^{\circ} \mathrm{C}$, twice with $\mathrm{KCl} 150 \mathrm{mM}$, and twice wi th MSHE buffer without BSA. 
The protein load was confirmed with an anti VDAC-1 antibody (1:700) (Santa Cruz Biotechnology INC.) ${ }^{13}$ and an anti $\beta$-actin antibody (1:1000) (Millipore) used as markers for outer mitochondrial membrane and cytosolic fraction, respectively. Removing the possibility that the mitochondrial fraction was contaminated, Western blot was performed where mitochondrial and cytosolic fractions were seeded and incubated with $\beta$-actin. As shown in Figure 1 Panel A, we used calreticulin antibody (1.1500) (Pierce) as an endoplasmic reticulum marker ${ }^{14}$. Calreticulin markings were observed in the cytosolic fraction, but were not observed in the mitochondrial fraction, discarding the possibility of contamination with endoplasmic reticulum. In Panel B, in the lanes where mitochondrial fractions were seeded, there is no evidence of the presence of $\beta$-actin,-ruling out the possible contamination of the cytosolic fraction. To determine mitochondrial purity, two mitochondrial specific antibodies were used: TOM40 antibody ${ }^{15}$ (1:1000) (Santa Cruz) and VDAC $^{13}$. TOM40 was included in Panel C (Figure 1), and it shows positive marking only in the mitochondrial fraction lanes. These findings, and the fact that VDAC was used as a loading control for the mitochondrial fractions, support the fact that the separation of the mitochondrial fraction was adequate.

$\underline{\text { Western Blot }}$

Protein samples from mitochondrial fractions $(30 \mu \mathrm{g})$ were separated by SDSPAGE and transferred to a nitrocellulose membrane. The samples were then incubated for one hour at room temperature in a blocking buffer containing $5 \%$ non fat milk and $5 \%$ BSA. Blots were then incubated overnight at $4^{\circ} \mathrm{C}$ with rabbit polyclonal anti-GSK-3 $\beta$ $(1: 1,000)$ (Santa Cruz Biotechnology INC.), mouse monoclonal anti-Ser9 phospho GSK-3 $\beta$ (1:300) (Millipore), rabbit monoclonal anti-Akt $(1: 1,000)$ (Cell Signaling Technology) and rabbit monoclonal anti-Ser473 phospho Akt antibodies $(1: 1,000)$ (Cell Signaling Technology). The blots were then incubated with goat anti-rabbit $(1: 5,000)$ and goat antimouse antibodies $(1: 3,000)$ (Millipore) at room temperature for 1 hour. Afterwards, they were rinsed with TBST. Blots were developed using the enhanced chemiluminescence method (BioRad) according to the manufacturer's instructions. Relative levels of GSK-3 $\beta$, phospho GSK-3 $\beta$, Akt and phospho Akt, were quantified by densitometric analysis using Image Gauge 4.0 software (Fujifilm). 


\section{Statistical Analysis}

Data are expressed as mean \pm standard error of the mean (SEM). Inter-group comparisons were carried out using analysis of variance (one way-ANOVA) followed by ttests with the $p$ value adjusted for multiple comparisons using the Bonferroni test. The data comparisons were not significant unless the corresponding $p$ value was less than $0.05 / k$, where $\mathrm{k}$ represents the number of comparisons.

\section{RESULTS}

Table 1 shows the behavior of the CPP and the infarct size following the administration of different concentrations of phenylephrine. Concentrations of 5 and $50 \mu \mathrm{M}$ produced a significant increase in CPP at $30 \mathrm{~min}$ of reperfusion, and an increase in the infarct size in both groups compared to group $\mathrm{I} / \mathrm{R}$. This is the reason why we administered a lower concentration of phenylephrine $(50 \mathrm{nM})$ where we could evidence a protective effect on the infarct size ( $p<0.05$ vs. $I / R$ ) without significant changes in CPP at 30 minutes of reperfusion.

Table 2 shows the left ventricular developed pressure (LVDP), the LVEDP and the CPP values at baseline and during different reperfusion times. In all groups, LVDP, a contractility index, was significantly lower compared with the respective pre-ischemic values, but showed no significant differences among groups throughout the procedure. Regarding myocardial stiffness, represented by LVEDP, a significant increase at 30 min of reperfusion without differences among the groups was observed. Phenylephrine is a selective $\alpha 1-A R s$ agonist and potent vasoconstrictor. We'd like to point out that the CPP values where phenylephrine was administered were similar to the other groups.

Figure 2 shows infarct size after 30 minutes of global no-flow ischemia and $120 \mathrm{~min}$ of reperfusion, expressed as a percentage of the total left ventricular area. In Panel A infarct size was $58.1 \pm 3.9 \%$ in the $\mathrm{l} / \mathrm{R}$ group. Ischemic postconditioning significantly decreased infarct size to $40.1 \pm 2.9 \%$ ( $p<0.05$ vs. I/R group). The administration of prazosin during the postconditioning protocol abolished the protective effect of postconditioning (63.5 $\pm 3.4 \%, p<0.05$ vs. postcon group). The infarct size in the group treated with prazosin 
alone during the first 2 minutes of reperfusion was similar to that observed in the I/R group $(58.8 \pm 3.6 \%)$, ruling out any possible effect of the drug per se. On the other hand, the activation of the $\alpha 1-A R s$ with phenylephrine during reperfusion significantly reduced the infarct size compared with the I/R group (35.3 $\pm 5.5, p<0.05$ vs. I/R). On the contrary, the activation of $\alpha 2-A R s$ by the administration of clonidine during the first two minutes of reperfusion did not modify the infarct size compared with the I/R group (55.4 $\pm 2.7 \%)$, demonstrating that $\alpha 2-A R s$ do not mediate protection. Panel B shows representative slices of the different experimental groups.

Figure 3 shows cardiac Akt protein expression (Panel A and C) and phosphorylation (Panel B and D) in the cytosolic fraction. Cardiac Akt protein expression was displayed as a percentage of the normoxic group. There were no significant changes in cytosolic Akt protein expression; however, ischemic postconditioning induced a significant increase in Akt phosphorylation compared with the I/R and postcon + prazosin groups (Panel B). The activation of $\alpha 1-A R s$, when phenylephrine was administered during reperfusion, shows a significant increase in Akt phosphorylation compared to I/R group, demonstrating that $\alpha 1-A R s$ would be involved in the protection. When we administered prazosin without ischemic postconditioning, we did not detect any changes compared with I/R group in the the cytosolic fraction (Panel D).

Figure 4 shows cardiac GSK-3 $\beta$ protein expression in the mitochondrial fraction. Cardiac GSK-3 $\beta$ mitochondrial protein expression was shown as a percentage of the normoxic group. There were no differences among groups in total cardiac GSK-3 $\beta$ protein expression (Panel $A$ and $C$ ). In Panel $B$ we show the normoxic, I/R, postconditioning, and postconditioning + prazosin groups. Ischemic postconditioning induced significant GSK-3 $\beta$ phosphorylation compared with the I/R group. Administration of prazosin during the ischemic postconditioning protocol decreased protein phosphorylation. In Panel $D$, we observed that prazosin without ischemic postconditioning did not modify GSK-3 $\beta$ phosphorylation compared with the I/R group. However a1-AR activation with phenylephrine administration increased GSK-3 $\beta$ phosphorylation compared with the I/R group, reaching similar values to the normoxic group. This finding suggests a link between mitochondrial GSK-3 $\beta$ and $\alpha 1-A R$ activation. 


\section{DISCUSSION}

The beneficial effect on infarct size was abolished by prazosin administration during the postconditioning protocol. The phenylephrine administration, an a1-ARs agonist, mimic the postconditioning effect and protected the heart against ischemia/reperfusion injury. Also, the activation of $\alpha 1-A R s$ involves Akt and GSK-3 $\beta$ phosphorylation. Therefore the results of the present study demonstrate that a1-ARs triggers the protective effect of postconditioning in the model of isolated rat hearts used herein.

As we mentioned, ischemic postconditioning and phenylephrine increased Akt phosphorylation in the cytosol. Given that GSK-3 $\beta$ is a downstream substrate of $A k t^{16}$ and is able to phosphorylate it, the present study sought to analyze the expression/phosphorylation state of this enzyme. The obtained results showed that GSK$3 \beta$ mitochondrial phosphorylation is increased in isolated rat hearts subjected to ischemic postconditioning and also in the presence of phenylephrine. In addition, it is noteworthy that administration of prazosin alone, without the postconditioning protocol, had no effect on infarct size, ruling out any possible effect of the drug per se.

Previous works used phenylephrine ${ }^{17,18}$ as a pharmacological tool with the aim of blocking a1-ARs in higher concentrations than the ones we used in the present research. An important difference is that in those studies, phenylephrine was administered before the ischemia period, and when trying to use the $50 \mu \mathrm{M}$, according to previous works ${ }^{17,18}$, we evidenced a powerful vasocontrictor effect. This, added to the fact that we administered it 30 minutes after ischemia, produced a significant increase on the infarct size and the CPP values (Table 1). Therefore, we performed experiments with different decreasing concentrations of phenylephrine, until we obtained a concentration that did not produce significant changes in the CPP behavior compared with the I/R protocol. We were able to evidence in this way a protective effect on the infarct size.

There is evidence supporting the hypothesis that $\alpha 1-A R$ stimulation is an important mechanism in the protection conferred by ischemic preconditioning ${ }^{6,7}$. A number of authors found that ischemic preconditioning and postconditioning share intracellular mechanisms, particularly during early reperfusion ${ }^{19,9}$. In this regard, stimulation of any $\mathrm{Gi}$ 
protein-coupled receptor has been shown to trigger preconditioning ${ }^{19}$, and a similar involvement of $G$ protein-coupled receptors is being revealed for postconditioning. The present study contributes to the understanding of the mechanisms involved in postconditioning due to the fact that the blockade of a1-ARs during ischemic postconditioning modulates infarct size- This suggests that these receptors may trigger the cardioprotection exerted by ischemic postconditioning. Consistent with our hypothesis, the lack of protection on the infarct size after the activation of a2-ARs with clonidine shows that protection would not be conferred by the activation of a2-ARs. Therefore, we are utilizing all this data and, as it was mentioned, even though we are not showing in a direct way the activation of $\alpha 1$-ARs, we strongly suggest that these receptors take part in the postconditioning protective mechanism, at least on isolated rat hearts.

The PI3K-Akt pathway is activated in response to the activation of a wide range of receptors, including G-protein-coupled receptors ${ }^{19}$. PI3K-Akt participates in different cellular processes by phosphorylating diverse substrates, including GSK-3 $\beta$ (glycogen and protein metabolism) ${ }^{19}$. Tsang et al. ${ }^{16}$ demonstrated that ischemic postconditioning protects the isolated rat heart by activating the PI3K-Akt pathway at the time of myocardial reperfusion. Different studies have suggested that Akt may protect the myocardium against ischemia/reperfusion injury by acting at the level of the mitochondria. In this regard, Juhaszova et al. ${ }^{20}$ demonstrated that ischemic preconditioning protects myocytes by phosphorylating GSK-3ß, a downstream target of Akt. In the present study, we showed that ischemic postconditioning phosphorylates Akt in the cytosol and GSK-3 $\beta$ in the mitochondria. Regarding $\alpha 1-$ ARs in myocardial ischemic protection, Zhao et al. ${ }^{21}$ demonstrated the protective effect of preconditioning in rats over-expressing a1-ARs compared to non-transgenic rats as it can be evidenced by a reduction on infarct size. According to the authors, this protective effect involves an increase in extracellular signalregulated kinases (ERK), the mitogen-activated protein kinase (MEK) phosphorylation, and also the inducible nitric oxide syntase (iNOS) activity and expression. Their findings are in agreement with the present study's results showing that blockade of $\alpha 1-A R$ receptors abolishes the protective effect of ischemic postconditioning. The latter involves Akt and GSK-3 $\beta$, which together with ERK, MEK and iNOS constitute the RISK-pathway, a group of proteins that activate during reperfusion and that are involved in the protection elicited 
by ischemic postconditioning ${ }^{22,9}$. In concordance, our results showed that $\alpha 1-A R s$ activation with phenylephrine increased Akt and GSK-3 $\beta$ phosphorylation.

It is known that GSK-3 $\beta$ contributes to different cellular processes including ischemia/reperfusion injury ${ }^{23}$. In contrast to many protein kinases, GSK-3 $\beta$ is active in resting cells and is inactivated by phosphorylation ${ }^{21}$. Inhibition of GSK-3 $\beta$ has been shown to reduce apoptosis and enhance cell survival, providing a plausible mechanism by which phosphorylation and inhibition of GSK-3 $\beta$ might mediate cardioprotection ${ }^{24}$. In this regard, Tong et al. showed that ischemic preconditioning reduces infarct size by increasing GSK$3 \beta$ phosphorylation through a PI3K-Akt dependent pathway ${ }^{10}$. Also Gomez et al. demonstrated that serine 9 phosphorylation of GSK-3 $\beta$ is required for cardioprotection by postconditioning, and it's likely to act by inhibiting the opening of the MPTP at the time of reperfusion in a transgenic mouse model of reperfused myocardial infarction ${ }^{11}$. In concordance with the fact that GSK-3 $\beta$ has a cardioprotective effect, Terashima et al. ${ }^{24}$ demonstrated that the GSK-3 $\beta$ phosphorylation, at the time of reperfusion by a Protein Kinase C- $\varepsilon(P K C-\varepsilon)$, PI3K-Akt and adenosine A2b receptor-dependent mechanism, contributes to the prevention of myocardial necrosis by pre-ischemic activation of the $\mathrm{K}^{+}$ mitochondrial channel. Also, it's important to consider the role of matrix metalloproteinases, particularly type 2 (MMP-2), since it's known that the increase of oxidative stress that occurs during reperfusion ${ }^{25}$ is one of the main triggers for the activation of this enzyme, and that both preconditioning ${ }^{26}$ and postconditioning ${ }^{27}$ decrease its activity. This is a fact that could partially explain the mechanisms of cardioprotection. In relation to this concept, Kandasamy $A D$ and Schulz $R$ demonstrated that MMP-2 activates GSK3-beta by proteolytic removal of the N-terminal domain where the inhibitory phosphorylation of Serine 9 (Ser-9) occurs ${ }^{28}$. Hence, this MMP-2-mediated increase of GSK-3 $\beta$ kinase activity may contribute to myocardial injury observed as a result of oxidative stress. Therefore we cannot discard that inhibition of MMP-2 by postconditioning could contribute by exerting its cardioprotective effect indirectly, given that the GSK-3 $\beta$ activity in a cell is dynamically regulated in response to stress.

The results of the present study demonstrate that activation of $\alpha 1-A R s$ induced phosphorylation of Ser9-GSK-3 $\beta$, since administration of prazosin during the ischemic postconditioning protocol decreased phospho-Ser9-GSK-3 $\beta$ expression and phenylephrine 
increased phospho-Ser9-GSK-3 $\beta$ expression. Ballou et al. ${ }^{29}$ demonstrated that the $\alpha 1-A R s$ can modulate GSK-3 $\beta$ activity in Rat- 1 cells in two ways. One mechanism antagonizes insulin-induced Ser-9 phosphorylation to cause an increase in GSK-3 $\beta$ activity, and the second promotes phosphorylation of GSK-3 $\beta$ at Ser-9 to cause a decrease in GSK-3 $\beta$ activity. In agreement with our findings, Zhai et al. ${ }^{30}$ demonstrated a differential role of GSK-3 $\beta$ during myocardial ischemia/reperfusion. The authors showed that activation of GSK-3 $\beta$ is detrimental during reperfusion but protective during ischemia. Activation of GSK-3 $\beta$ during ischemia and inactivation of GSK-3 $\beta$ during reperfusion are both compensatory for the heart. Ischemic postconditioning acting in the reperfusion phase induces GSK-3 $\beta$ phosphorylation, decreasing its activity and conferring cardioprotection.

In the present study, ischemic postconditioning and phenylephrine administration reduced infarct size but were unable to improve ventricular function (contractile state and myocardial stiffness). The absence of improvement of left ventricular function in our study may be due to the presence of myocardial stunning areas peripheral to the infarct zone. In this sense, it has been well shown that the presence of a certain degree of postischemic dysfunction (stunned myocardium) reverts approximately after 48/72 hrs of reperfusion. Therefore the change on infarct size in acute experiments does not significantly influence the ventricular function ${ }^{31}$.

In conclusion, the data obtained herein suggest that ischemic postconditioning decreases infarct size by activation of the $\alpha 1-A R$ pathway, which could involve Akt and GSK-3 $\beta$ phosphorylation. These current results could indicate that after being

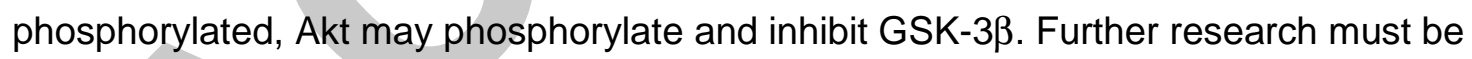
conducted to better understand the mechanism of ischemic postconditioning cardioprotection, and to make it fully applicable in the clinical setting.

\section{ACKNOWLEDGMENTS}

This work was supported by University of Buenos Aires grant (UBACYT B069), National Agency of Scientific and Technological Promotion (08/PICT0258). National Council of Scientific and Technological Research (CONICET, PIP 11/0197). We want to thank Dr. Ignacio Cebrián for giving us antibodies to perform the Western Blots related to cytosolic and mitochondrial purity isolation. 


\section{REFERENCES}

1. Murry CE, Jennings RB, Reimer KA. Preconditioning with ischemia: a delay of lethal cell injury in ischemic myocardium. Circulation 1986; 74: 1124-1136.

2). Zhao ZQ, Vinten-Johansen J. Postconditioning: reduction of reperfusion-induced injury. Cardiovasc Res.2006; 70:200-211.

3. Donato M, D'Annunzio V, Berg G, Gonzalez G, Schreier L, Morales C, Wikinski RL, Gelpi RJ Ischemic postconditioning reduces infarct size by activation of A1 receptors and K+(ATP) channels in both normal and hypercholesterolemic rabbits. J. Cardiovasc Pharmacol. 2007; 49(5):287-92.

4. Xi L, Das A, Zhao ZQ, Merino VF, Bader M, Kukreja RC (2008). Loss of myocardial ischemic postconditioning in adenosine A1 and bradykinin B2 receptors gene knockout mice. Circulation. 2008; 118: S32-S37.

5. Pateliya BB, Singh N, Jaggi AS. Possible role of opioids and KATP channels in neuroprotective effect of postconditioning in mice. Biol Pharm Bull. 2008; 31: 1755-1760.

6. Gao H, Chen L, Yang HT. Activation of alpha1-adrenoceptors alleviates ischemia/reperfusion injury by limitation of mitochondrial $\mathrm{Ca} 2+$ overload in cardiomyocytes. Cardiovasc Res. 2007;75:584-595.

7. Banerjee A, Locke-Winter C, Rogers KB, Mitchell MB, Brew EC, Cairns CB, Bensard DD, Harken AH. Preconditioning against myocardial dysfunction after ischemia and reperfusion by an alpha 1-adrenergic mechanism. Circ Res. 1993; 73: 656-670. 
8. Vasara E, Seraskeris S, Lazou A. Activation of alpha 1-adrenoceptors is not essential for the mediation of ischaemic preconditioning in rat heart. Clin Exp Pharmacol Physiol. 2002; 29: 11-17.

9. Hausenloy DJ, Yellon DM. Survival kinases in ischemic preconditioning and postconditioning.Cardiovasc Res. 2006; 70: 240-253.

10. Tong H, Imahashi K, Steenbergen C, Murphy E. Phosphorylation of glycogen synthase kinase-3beta during preconditioning through a phosphatidylinositol-3-kinase--dependent pathway is cardioprotective. Circ Res. 2002; 90: 377-379.

11. Gómez L, Paillard M, Thibault H, Derumeaux G, Ovize M. Inhibition of GSK3beta by postconditioning is required to prevent opening of the mitochondrial permeability transition pore during reperfusion. Circulation. 2008; 117: 2761-2768.

12. Inserte J, García-Dorado D, Hernando V, Soler-Soler J. Calpain-mediated impairment of $\mathrm{Na}+/ \mathrm{K}+-\mathrm{ATP}$ ase activity during early reperfusion contributes to cell death after myocardial ischemia. Circ Res. 2005; 97: 465-473.

13. Salnikov V, Lukyánenko Y O, Frederick C A, Lederer W J, Lukyánenko V. Probing the Outer Mitochondrial Membrane in Cardiac Mitochondria with Nanoparticles. Biophys J. 2007; 92(3): 1058-1071.

14. George I, Sabbah HN, Xu K, Wang N, Wang J. $\beta$-adrenergic receptor blockade reduces endoplasmic reticulum stress and normalizes calcium handling in a coronary embolization model of heart failure in canines. Cardiovasc Res. 2011;91(3):447-55. 
15. Eura $\mathrm{Y}$, Ishihara N, Oka T, Mihara K. Identification of a novel protein that regulates mitochondrial fusion by modulating mitofusin (Mfn) protein function. J Cell Sci. 2006; 119(Pt 23):4913-25

16. Tsang A, Hausenloy DJ, Mocanu MM, Yellon DM. Postconditioning: a form of "modified reperfusion" protects the myocardium by activating the phosphatidylinositol 3kinase-Akt pathway. Circ Res. 2004; 95(3): 230-232.

17. Naderi R, Imani A, Faghihi M. Phenylephrine produces late pharmacological preconditioning in the isolated rat heart. Eur J Pharmacol. 2010; 627(1-3):203-8.

18. Tsuchida A, Liu Y, Liu GS, Cohen MV, Downey JM. alpha 1-adrenergic agonists precondition rabbit ischemic myocardium independent of adenosine by direct activation of protein kinase C. Circ Res. 1994; 75: 576-585

19. Vinten-Johansen J, Zhao ZQ, Jiang R, Zatta AJ, Dobson GP. Preconditioning and postconditioning: innate cardioprotection from ischemia-reperfusion injury. J Appl Physiol. 2007; 103: 1441-1448.

20. Juhaszova M, Zorov DB, Yaniv Y, Nuss HB, Wang S, Sollott SJ. Role of glycogen synthase kinase-3beta in cardioprotection.Circ Res. 2009; 104: 1240-1252.

21. Zhao X, Park J, Ho D, Gao S, Yan L, Ge H, lismaa S, Lin L, Tian B, Vatner DE, Graham RM, Vatner SF. Cardiomyocyte overexpression of the $\alpha 1 \mathrm{~A}$-adrenergic receptor in the rat phenocopies second but not first window preconditioning. Am J Physiol Heart Circ Physiol. 2012; 302(8):H1614-24. 
22. Hausenloy DJ, Tsang A, Yellon DM. The reperfusion injury salvage kinase pathway: a common target for both ischemic preconditioning and postconditioning. Trends Cardiovasc Med. 2005; 15: 69-75.

23. McLean BA, Kienesberger PC, Wang W, Masson G, Zhabyeyev P, Dyck JR, Oudit GY. Enhanced recovery from ischemia-reperfusion injury in PI3Ka dominant negative hearts: investigating the role of alternate PI3K isoforms, increased glucose oxidation and MAPK signaling. J Mol Cell Cardiol. 2013; 54: 9-18.

24. Terashima Y, Sato T, Yano T, Maas O, Itoh T, Miki T, Tanno M, Kuno A, Shimamoto K, Miura T. Roles of phospho-GSK-3 $\beta$ in myocardial protection afforded by activation of the mitochondrial K ATP channel. J Mol Cell Cardiol. 2010; 49:762-70.

25. Wang W, Sawicki G, Schulz R. Peroxynitrite-induced myocardial injury is mediated through matrix metalloproteinase-2. Cardiovasc Res. 2002; 53:165-74.

26. Lalu MM, Csonka C, Giricz Z, Csont T, Schulz R, Ferdinandy P. Preconditioning decreases ischemia/reperfusion-induced release and activation of matrix metalloproteinase-2. Biochem Biophys Res Commun. 2002; 296: 937-41.

27. Donato M, D'Annunzio V, Buchholz B, Miksztowicz V, Carrión CL, Valdez LB, Zaobornyj T, Schreier L, Wikinski R, Boveris A, Berg G, Gelpi RJ. Role of matrix metalloproteinase-2 in the cardioprotective effect of ischaemic postconditioning. Exp Physiol. 2010; 95 :274-81. 
28. Kandasamy AD, Schulz R. Glycogen synthase kinase-3beta is activated by matrix metalloproteinase-2 mediated proteolysis in cardiomyoblasts. Cardiovasc Res. 2009; 83: 698-706.

29. Ballou LM, Tian PY, Lin HY, Jiang YP, Lin RZ. Dual regulation of glycogen synthase kinase-3beta by the alpha1A-adrenergic receptor. J BiolChem. 2001; 276: 40910-40916.

30. Zhai P, Sciarretta S, Galeotti J, Volpe M, Sadoshima J. Differential Roles of GSK-3ß during Myocardial Ischemia and Ischemia/Reperfusion. Circ Res. 2011; 109: 502-511.

31. Cohen MV, Yang XM, Downey JM. Smaller infarct after preconditioning does not predict extent of early functional improvement of reperfused heart. Am J Physiol. 1999; 277(5 Pt 2):H1754-61 


\section{LEGENDS}

Figure 1: Panel A: Shows the calreticulin representative blot in mitochondrial and cytosolic fractions. This figure shows that the mitochondrial fraction was free from contamination with endoplasmic reticulum. Panel B: $\beta$-actin representative blot in mitochondrial fraction and cytosolic fraction. This figure is showed to discard mitochondrial contamination with cytosolic fraction. Panel C: TOM40 representative blot in mitochondrial and cytosolic fraction. This figure shows that TOM40, a mitochondrial membrane protein, is only present in the mitochondrial fraction, confirming its correct isolation. Nx: normoxic group; I/R: ischemia/reperfusion group; Postcon: Ischemic postconditioning group; PE: Phenylephrine group.

Figure 2: Infarct size. Panel A shows infarct size expressed as a percentage of the total left ventricular area. Infarct size decreased significantly with ischemic postconditioning, and ischemic postconditioning + prazosin treatment abolished this beneficial effect. Administration of prazosin or clonidine alone, without postconditioning, did not modify infarct size, but phenylephrine administration reduced infarct size. Panel B shows representative slices of the different experimental groups. * $p<0.05$ vs I/R, Postcon, Postcon + prazosin and prazosin. I/R: Ischemia/reperfusion group; Postcon: Ischemic postconditioning group; PE: Phenylephrine group; CL: Clonidine

Figure 3: Akt protein. Akt protein expression (Panels $A$ and $C$ ) and phosphorylation (Panels $B$ and $D$ ) in the cytosolic fraction are represented. There were no significant changes in cytosolic Akt protein expression (Panels $A$ and $C$ ). However, ischemic postconditioning induced significant Akt phosphorylation which was blunted by postcon + prazosin (Panel B). Panel D showed that phenylephrine administration increased Akt phosphorylation, but prazosin alone did not modify Akt phosphorylation. Cardiac Akt protein expression and phosphorylation were expressed as a percentage of the normoxic group (Nx). Equal protein loading was confirmed by $\beta$ actin. * $p<0.05$ vs Nx and Postcon; 
\# $p<0.05$ vs. Nx and PE. I/R: Ischemia/reperfusion group; Postcon: Ischemic postconditioning group; PE: Phenylephrine group.

Figure 4: Glycogen synthasa kinase $3 \beta$ protein. Cardiac GSK-3 $\beta$ protein expression (Panels $A$ and $C$ ) and phosphorylation (Panels $B$ and $D$ ) in the mitochondrial fractions are represented. GSK-3 $\beta$ mitochondrial protein expression and phosphorylation were expressed as a percentage of the normoxic group $(\mathrm{Nx})$. There were no differences among the groups in total cardiac GSK-3 $\beta$ protein expression (Panels $A$ and $C$ ). Ischemic postconditioning induced significant GSK-3 $\beta$ phosphorylation compared with the I/R group, but administration of prazosin during the ischemic postconditioning protocol decreased protein phosphorylation (Panel B). Phenylephrine administration increased GSK-3 $\beta$ phosphorylation, but prazosin alone did not change GSK-3 $\beta$ phosphorylation compared with $\mathrm{I} / \mathrm{R}$ (Panel $\mathrm{D}$ ). Equal protein loading was confirmed by VDAC in the mitochondrial fraction. ${ }^{*} p<0.05$ vs. Nx and Postcon; \# $p<0.05$ vs. Nx and PE. I/R: Ischemia/reperfusion group; Postcon: Ischemic postconditioning group; PE: Phenylephrine group; Nx: Normoxic group. 
Table 1: Response of different concentrations of Phenylephrine administration on infarct size and coronary perfusion pressure (CPP)

\begin{tabular}{|c|c|c|c|}
\hline Groups & Baseline CPP (mmHg) & 30 min R CPP (mmHg) & $\begin{array}{c}\text { Infarct size } \\
(\%)\end{array}$ \\
\hline $\mathbf{I} / \mathbf{R}$ & $66.1 \pm 1.1$ & $102.3 \pm 3.1^{*}$ & $58.1 \pm 3.9$ \\
\hline PE $50 \mu \mathrm{M}$ & $67.9 \pm 0.9$ & $152.8 \pm 13.5^{* \&}$ & $82.5 \pm 5.3^{\&}$ \\
\hline PE $12.5 \mu \mathrm{M}$ & $67.8 \pm 3.3$ & $145.5 \pm 9.6^{* \&}$ & $74.4 \pm 6.2^{\&}$ \\
\hline PE $50 \mathrm{nM}$ & $63.1 \pm 3.2$ & $103.4 \pm 7.3^{*}$ & $35.3 \pm 5.5^{\#}$ \\
\hline
\end{tabular}


Table 2: Left ventricular function and coronary perfusion pressure.

\begin{tabular}{|c|c|c|c|c|c|}
\hline & Groups & Baseline & 2' Rep & 15' Rep & 30' Rep \\
\hline \multirow{6}{*}{$\begin{array}{c}\text { LVDP } \\
(\mathrm{mmHg})\end{array}$} & I/R & $101.6 \pm 3.6$ & $15.8 \pm 3.1^{*}$ & $11.3 \pm 3.9^{*}$ & $12.6 \pm 2.6^{\star}$ \\
\hline & Postcon & $105.4 \pm 4.7$ & $9.1 \pm 1.4^{*}$ & $7.4 \pm 1.4^{*}$ & $11.4 \pm 1.8^{*}$ \\
\hline & Postcon + Prazosin $1 \mu \mathrm{M}$ & $103.9 \pm 4.3$ & $8.1 \pm 1.2^{*}$ & $7.6 \pm 0.8^{*}$ & $10.3 \pm 2.3^{*}$ \\
\hline & Prazosin $1 \mu \mathrm{M}$ & $109.5 \pm 3.2$ & $15.4 \pm 6.1^{*}$ & $6.7 \pm 1.7^{*}$ & $9.9 \pm 2.5^{\star}$ \\
\hline & Phenylephrine $50 \mathrm{nM}$ & $102.1 \pm 2.8$ & $13.1 \pm 1.7^{*}$ & $17.2 \pm 4.9^{*}$ & $19.6 \pm 3.8^{*}$ \\
\hline & Clonidine $10^{-6} \mathrm{M}$ & $99.7 \pm 5.2$ & $14.2 \pm 8.5^{\star}$ & $18.9 \pm 5.2^{*}$ & $21.3 \pm 6.5^{\star}$ \\
\hline \multirow{6}{*}{$\begin{array}{l}\text { LVEDP } \\
(\mathrm{mmHg})\end{array}$} & $\mathrm{I} / \mathbf{R}$ & $9.4 \pm 0.3$ & 110. $\pm 4.8^{*}$ & $110.9 \pm 4.1^{*}$ & $104.7 \pm 4.5$ \\
\hline & Postcon & $7.8 \pm 0.4$ & $117.9 \pm 4.2^{*}$ & $121.2 \pm 4.2^{*}$ & $107.1 \pm 4.5^{\star}$ \\
\hline & Postcon + Prazosin $1 \mu \mathrm{M}$ & $7.3 \pm 0.4$ & $115.8 \pm 9.9^{*}$ & $118.9 \pm 7.7^{*}$ & $108.1 \pm 9.8^{*}$ \\
\hline & Prazosin $1 \mu \mathrm{M}$ & $7.6 \pm 0.8$ & $92.8 \pm 12.7^{*}$ & $126.4 \pm 12.2^{*}$ & $111.3 \pm 3.7^{*}$ \\
\hline & Phenylephrine 50nM & $7.9 \pm 0.8$ & $94.1 \pm 4.8^{*}$ & $104.1 \pm 5.9^{*}$ & $100.3 \pm 9.7^{*}$ \\
\hline & Clonidine $10^{-6} \mathrm{M}$ & $8.1 \pm 0.7$ & $99.8 \pm 5.9^{*}$ & $112.9 \pm 7.2^{*}$ & $107.8 \pm 8.6^{*}$ \\
\hline \multirow{6}{*}{$\underset{(\mathrm{mmHg})}{\mathrm{CPP}}$} & $\mathbf{I} / \mathbf{R}$ & $66.1 \pm 1.1^{*}$ & $87.1 \pm 2.3^{*}$ & $90.4 \pm 2.7^{*}$ & $102.3 \pm 3.1$ \\
\hline & Postcon & $67.9 \pm 0.9^{*}$ & $90.1 \pm 2.5^{*}$ & $89.9 \pm 2.7^{*}$ & $99.7 \pm 3.5^{\star}$ \\
\hline & Postcon + Prazosin $1 \mu \mathrm{M}$ & $67.8 \pm 3.3$ & $87.5 \pm 2.7^{\star}$ & $84.7 \pm 3.6^{*}$ & $91.8 \pm 9.3^{*}$ \\
\hline & Prazosin $1 \mu \mathrm{M}$ & $63.1 \pm 3.2$ & $85.8 \pm 4.1^{*}$ & $101.1 \pm 4.9^{*}$ & $106.1 \pm 11.8^{*}$ \\
\hline & Phenylephrine 50nM & $64.1 \pm 2.7$ & $83.3 \pm 4.5^{\star}$ & $89.3 \pm 3.2^{*}$ & $103.4 \pm 7.3^{*}$ \\
\hline & Clonidine $10^{-6} \mathrm{M}$ & $71.1 \pm 3.9$ & $89.5 \pm 6.2^{*}$ & $93.2 \pm 4.9^{*}$ & $101.8 \pm 8.9^{*}$ \\
\hline
\end{tabular}

LVDP: Left ventricular developed pressure; LVEDP: Left ventricular end diastolic pressure; CPP: Coronary perfusion pressure. I/R: Ischemia/Reperfusion group; Postcon: ischemic postconditioning. * $p<0.05$ vs baseline 

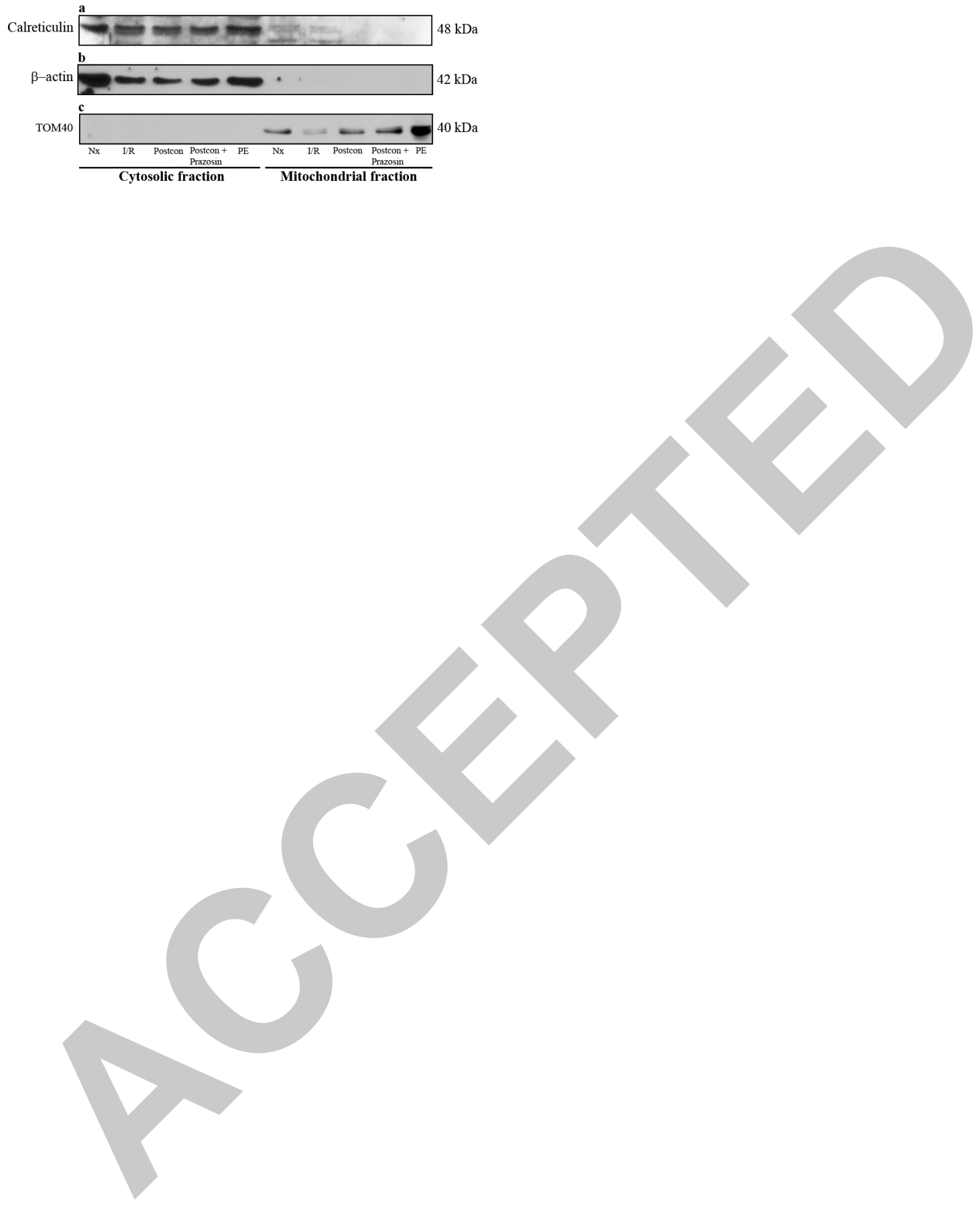

Copyright (c) Lippincott Williams \& Wilkins. All rights reserved. 

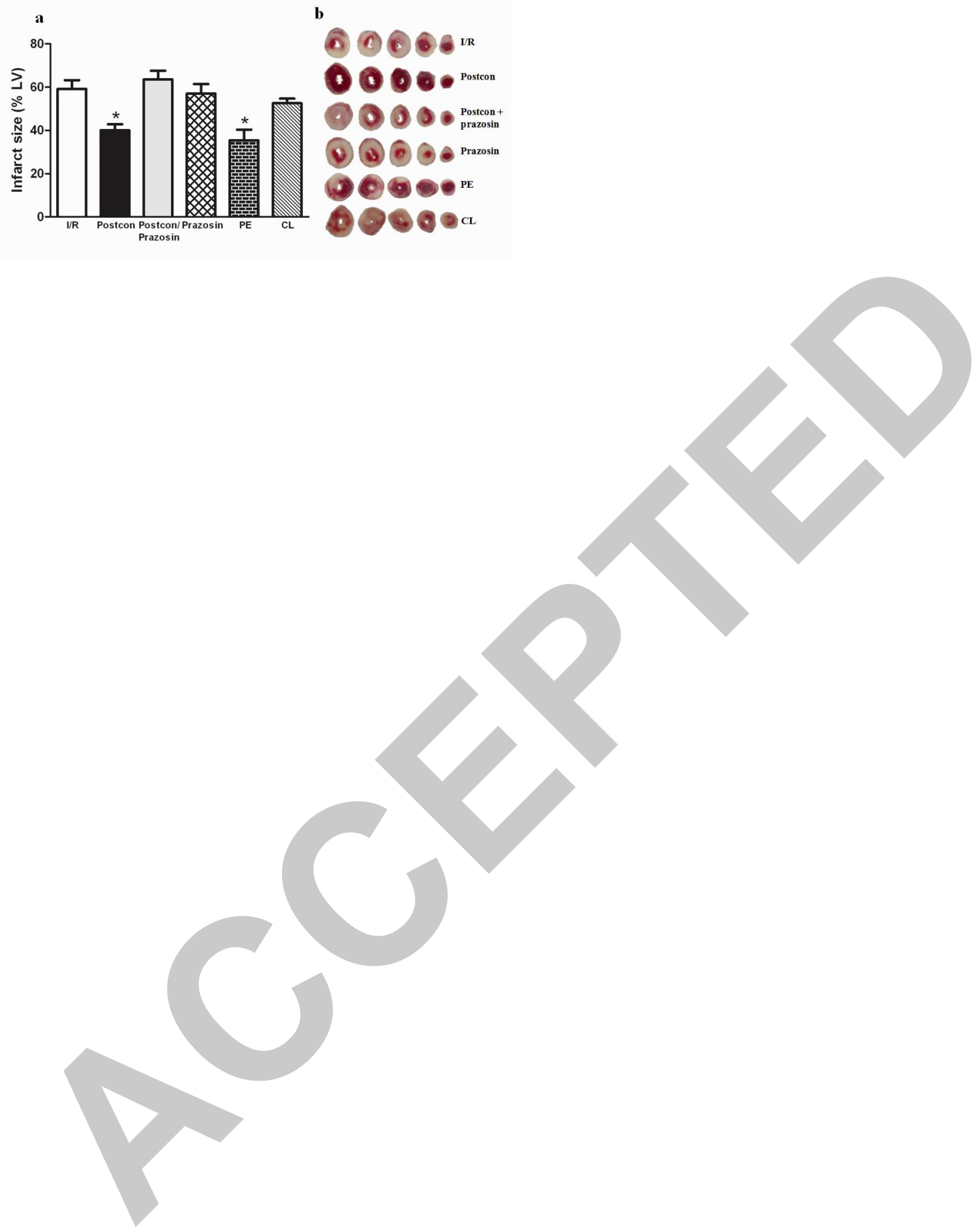

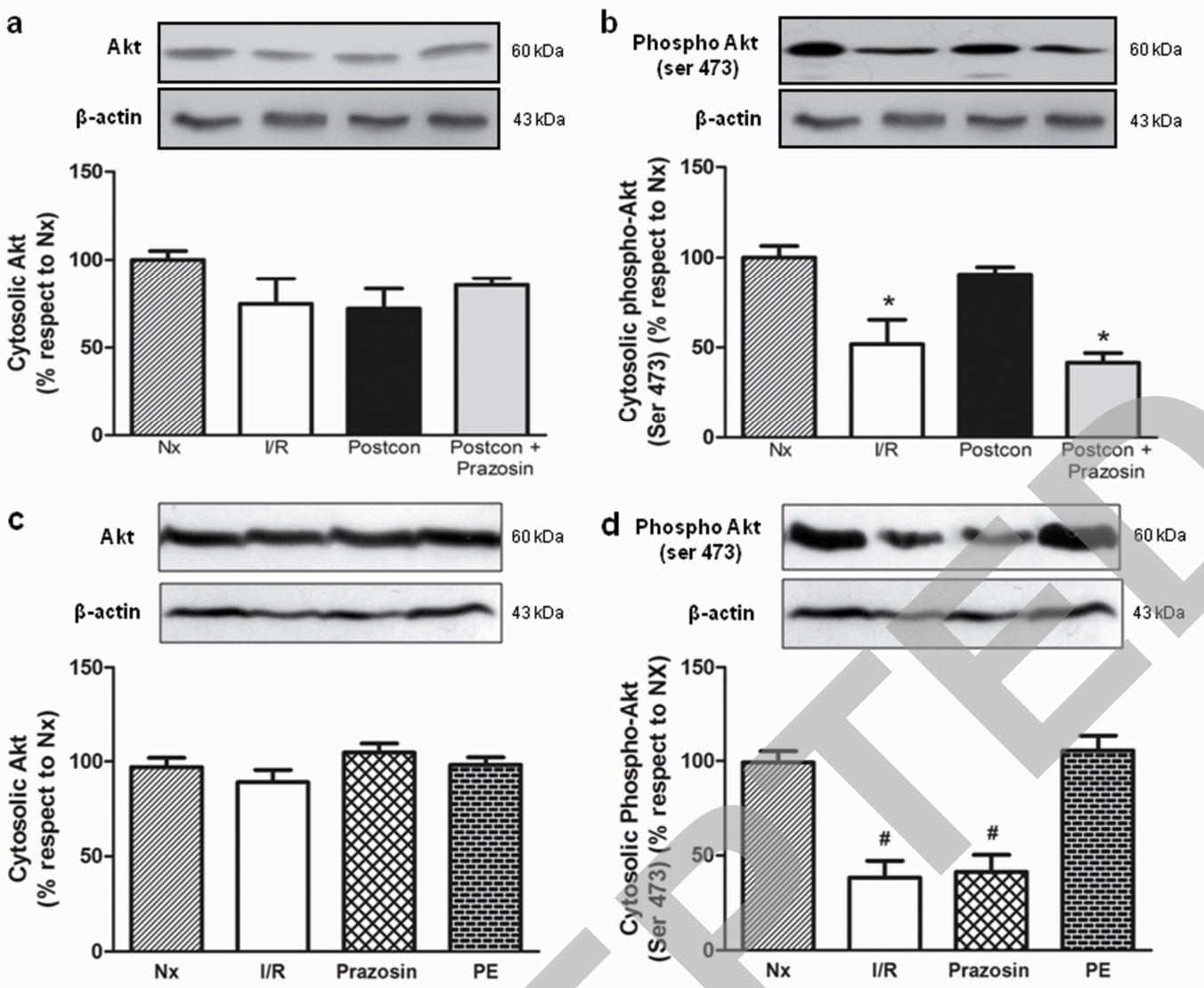

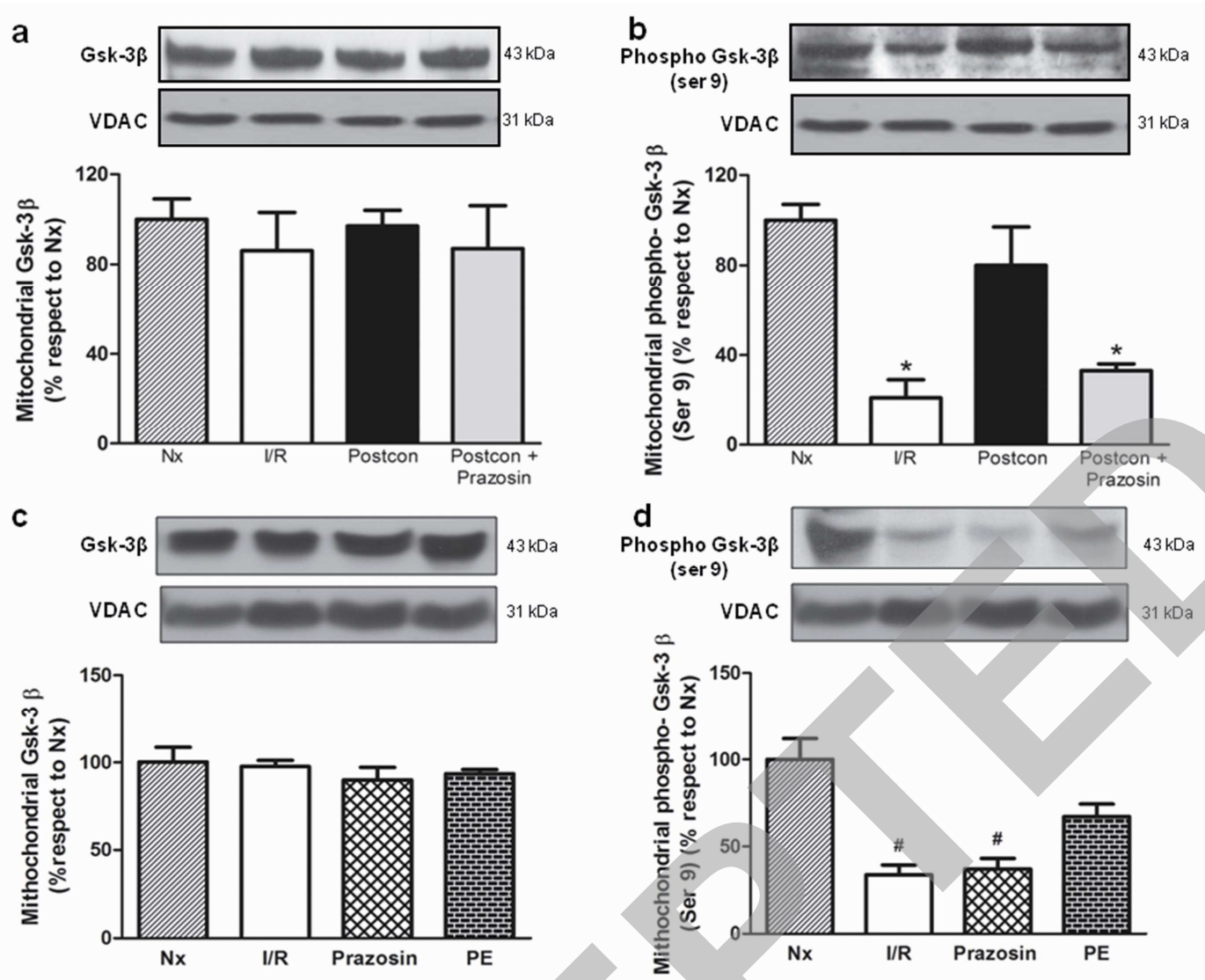